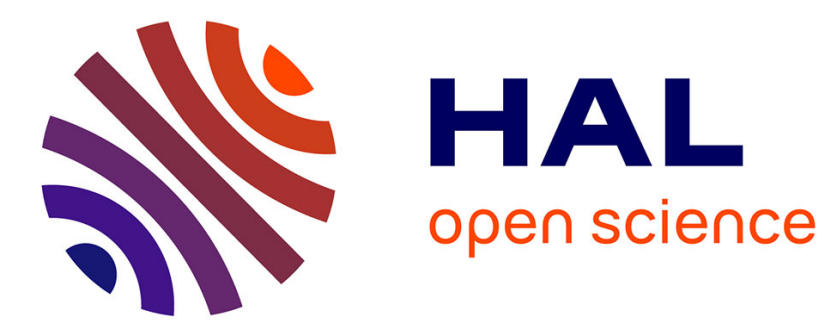

\title{
Effets non linéaires dans les fibres optiques. Applications
}

\author{
J.P. Pocholle, M. Papuchon, J. Raffy, C. Puech
}

\section{To cite this version:}

J.P. Pocholle, M. Papuchon, J. Raffy, C. Puech. Effets non linéaires dans les fibres optiques. Applications. Revue de Physique Appliquée, 1986, 21 (11), pp.673-681. 10.1051/rphysap:019860021011067300 . jpa-00245488

\section{HAL Id: jpa-00245488 https://hal.science/jpa-00245488}

Submitted on 1 Jan 1986

HAL is a multi-disciplinary open access archive for the deposit and dissemination of scientific research documents, whether they are published or not. The documents may come from teaching and research institutions in France or abroad, or from public or private research centers.
L'archive ouverte pluridisciplinaire HAL, est destinée au dépôt et à la diffusion de documents scientifiques de niveau recherche, publiés ou non, émanant des établissements d'enseignement et de recherche français ou étrangers, des laboratoires publics ou privés. 


\title{
Effets non linéaires dans les fibres optiques. Applications
}

\author{
J. P. Pocholle, M. Papuchon, J. Raffy et C. Puech \\ Thomson-CSF/LCR, B.P. 10, 91401 Orsay, France
}

\begin{abstract}
Résumé. - Une onde optique de forte intensité se propageant dans une fibre optique unimodale, peut générer un grand nombre d'effets non linéaires. Cet article analyse l'amplification optique directe fondée sur le développement de l'effet Raman stimulé, avec la perspective d'emploi dans les systèmes de communication par fibres optiques. Par ailleurs, la génération de nouvelles fréquences par mélange de fréquences optiques dans les fibres est présentée. Les applications potentielles liées à ces effets non linéaires sont également mentionnées.
\end{abstract}

\begin{abstract}
An intense light beam propagating through a single mode fiber can produce a wide variety of nonlinear phenomena. This paper discusses direct optical amplification of laser diode signal by stimulated Raman scattering, regarding applications in fiber optic communications. On the other hand, the generation of difference frequencies by three wave mixing of light are considered. Potential applications are also discussed.
\end{abstract}

\section{Introduction.}

Les structures optiques de type guide d'onde se prêtent bien à la génération et à l'exploitation d'effets non linéaires. De telles configurations présentent la particularité de conserver un fort pouvoir de confinement des champs associés aux ondes optiques sur de grandes distances, offrant par là même des longueurs d'interactions élevées.

Ces longueurs effectives d'interaction contribuant à la génération d'effets non linéaires sont cependant limitées, soit par le facteur d'affaiblissement, soit par la dispersion des paramètres optogéométriques. La maîtrise de ces paramètres liée au processus de fabrication ou au conditionnement des guides optiques autorise le développement de nouveaux systèmes ou composants réalisant les fonctions :

- génération d'harmoniques ou conversion de fréquences,

- amplification optique directe,

- sources accordables,

- remise en forme d'impulsions.

Déjà, dans les années 1960, avec l'avènement des sources cohérentes optiques, des structures de type guides optiques actifs étaient proposées [1] et réalisées [2]

Des opérations d'amplification optique de signaux issus d'une diode laser étaient observées en employant des fibres optiques à base de verres dopés d'ions $\mathrm{Nd}^{3+}$ [3]. L'emploi de telles fibres comme préamplificateur optique de signaux au niveau d'un récepteur était également démontré [4].
C'est par ailleurs dans ce cadre, que furent établies les principales relations régissant la propagation dans un milieu à deux diélectriques de configuration cylindrique $[5,6]$.

Bien plus tard, avec le développement de procédés de dépôts permettant l'obtention de fibres optiques à base de silice à faibles pertes en transmission $[7,8]$, l'adjonction d'ions de terres rares permettait de réaliser des sources miniatures pompées optiquement $[9,10]$. Aujourd'hui des efforts sont également menés pour réaliser des fibres optiques cristallines $\left(\mathrm{LiNbO}_{3}, \mathrm{YAG}, \mathrm{Rubis}, . ..\right)$ présentant des structures à symétrie de révolution tout en conservant les propriétés cristallines d'orientation, ce qui permet d'envisager la fabrication de fibres lasers [11-13].

Par ailleurs, la conservation des propriétés cristallines permet d'envisager la réalisation de modulateurs électrooptiques ou d'effectuer des opérations de doublage ou de conversion de fréquences à partir d'une onde émise par une diode laser [13] en bénéficiant du fort pouvoir de confinement associé à de telles structures.

Dans le même ordre d'idée, le développement de matériaux organiques présentant de fortes nonlinéarités optiques $[14,15]$ insérées dans des structures optiques de type guide d'onde devrait permettre de créer de nouveaux composants ouvrant la voie au traitement optique de l'information ou de réaliser des sources quasi cohérentes situées dans le domaine spectral $0,4 \mu \mathrm{m}$ à partir de diodes lasers opérant dans le proche IR. (Ex : composés semiconducteurs AlGaAs-GaAs émettant entre 0,8-0,9 $\mu \mathrm{m}$ ). 
Ainsi, les structures de type guides optiques permettent :

- d'accroître les longueurs d'interactions,

- d'augmenter le pouvoir de confinement,

- d'ajuster les conditions d'accord de phase en modifiant les paramètres optogéométriques des guides.

Le maniement ou la prise en compte de ces concepts d'optique guidée devrait donc permettre aux spécialistes des matériaux de valoriser ou de réactualiser leurs travaux.

Nous allons illustrer le développement d'effets non linéaires en propagation guidée, en considérant des structures de type fibres optiques à base de silice. Ce matériau est de classe centro-symétrique et seuls les effets non linéaires d'ordre impair et en particulier du troisième ordre peuvent prendre naissance.

Parmi ceux-ci, nous pouvons citer :

- l'effet Kerr ou le mélange à quatre photons,

- l'effet Brillouin,

- l'effet Raman.

De tels effets peuvent se développer dans les fibres optiques à base de silice bien que la silice ne présente pas de forts coefficients non linéaires en comparaison avec ceux observés dans certains liquides ou cristaux. Dans les fibres optiques à base de silice et plus particulièrement dans les fibres optiques unimodales, le paramètre définissant le pouvoir de confinement est relié à la dimension du mode ; celleci présente un diamètre compris entre 6 et $10 \mu \mathrm{m}$.

Ainsi, si l'on couple à cette fibre, une puissance crête de l'ordre du Watt, alors des densités de puissance de l'ordre de quelques $\mathrm{MW} / \mathrm{cm}^{2}$ peuvent être véhiculées sur de grandes distances pour peu que le guide présente un faible facteur d'affaiblissement. Or dans les fibres optiques à base de silice, les pertes en transmission dans la fenêtre spectrale 1$1,5 \mu \mathrm{m}$ sont comprises entre 1,2 et $0,2 \mathrm{~dB} / \mathrm{km}$. La longueur d'interaction en sera d'autant plus importante, rendant possible le développement d'effets non linéaires en régime stimulé.

Parmi ceux-ci, nous pouvons citer l'effet Raman permettant d'effectuer des opérations d'amplification optique-optique directe pour peu que l'écartement en fréquences entre l'onde dite de « pompe » et l'onde « signal » corresponde au décalage en fréquence Raman spécifique du matériau constituant le guide optique.

Nous allons décrire plus particulièrement dans cet article les interactions non linéaires du type effet Raman et mélange à quatre photons se développant dans les fibres optiques à base de silice. Des opérations d'amplification optique directe peuvent être réalisées à partir de l'effet Raman pour peu que l'écartement en fréquences entre l'onde dite de « pompe » et l'onde « signal » corresponde au décalage en fréquence Raman spécifique du matériau constituant le guide optique.

Le processus de mélange à quatre photons permet également de réaliser des opérations d'amplification ou de conversion de fréquences. De plus, en modifiant ou en contrôlant la température de la fibre, il est possible de réaliser une source accordable dans le proche infrarouge.

De telles applications sont donc l'objet du présent article.

\section{Polarisation non linéaire (rappel).}

La polarisation d'un milieu peut être représentée sous sa forme la plus générale par la relation :

$$
\mathbf{P}=\varepsilon_{0}\left(\chi^{(1)} \mathbf{E}+\chi^{(2)} \mathbf{E E}+\chi^{(3)} \mathbf{E E E}\right)
$$

où les coefficients $\chi^{(n)}$ relient le vecteur polarisation aux vecteurs champs électriques. Ces coefficients appelés susceptibilité linéaire et non linéaire sont des tenseurs d'ordre $n+1$.

Cette polarisation peut être décomposée selon une composante linéaire et une contribution non linéaire :

$$
\begin{aligned}
\mathbf{P} & =\mathbf{P}_{\mathrm{L}}+\mathbf{P}_{\mathrm{NL}} \\
\mathbf{P}_{\mathrm{L}} & =\varepsilon_{0} \chi^{(1)} \mathbf{E} \\
\mathbf{P}_{\mathrm{NL}} & =\varepsilon_{0}\left(\chi^{(2)} \mathbf{E E}+\chi^{(3)} \mathbf{E E E}+\cdots\right) .
\end{aligned}
$$

Dans le cas où le matériau est de type centrosymétrique, la susceptibilité non linéaire du second ordre est nulle $\left(\chi^{(2)}=0\right)$ et la susceptibilité non linéaire du $3^{\mathrm{c}}$ ordre est à l'origine d'effets non linéaires dans ces milieux. On peut décomposer le coefficient $\chi^{(3)}$ en partie réelle et imaginaire :

$$
\chi^{(3)}=\chi^{\prime(3)}-j \chi^{\prime(3)} \text {. }
$$

La partie imaginaire est alors associée à l'effet Raman alors que la partie réelle correspond en première approximation à la contribution électronique responsable des opérations de mélange de fréquences et de l'effet Kerr optique.

\section{Effet Raman stimulé. Amplification optique.}

3.1 RAPPEL THÉORIQUE. - L'effet Raman peut être décrit phénoménologiquement comme un processus de diffusion inélastique d'une onde optique par couplage avec les niveaux vibrationnels de l'édifice moléculaire constituant le milieu de propagation. Une approche quantique permet d'établir l'évolution du nombre de photons aux fréquences pompe et Stokes [16] :

$$
\begin{aligned}
& \frac{\mathrm{d} n_{\mathrm{p}}}{\mathrm{d} Z}+\alpha_{\mathrm{p}} n_{\mathrm{p}}=-\gamma_{\mathrm{s}} n_{\mathrm{p}}\left(n_{\mathrm{s}_{1}}+1+n_{\mathrm{s}_{2}}+1+\cdots+n_{\mathrm{s}_{q}}+1\right) \\
& \frac{\mathrm{d} n_{\mathrm{s} j}}{\mathrm{~d} Z}+\alpha_{\mathrm{s}} n_{\mathrm{s} j}=\gamma_{\mathrm{s}} n_{\mathrm{p}}\left(n_{\mathrm{s} j}+1\right) .
\end{aligned}
$$


$\gamma_{\mathrm{s}}$ correspond au facteur de gain Raman du milieu ; on considère dans ces équations que l'onde de pompe est localisée dans un mode longitudinal alors que le nombre de photons Stokes par mode longitudinal est représenté par le terme $n_{\mathrm{s} j}+1 . n_{\mathrm{s} j}$ est associé au nombre de photons injectés dans le mode $j$ et le terme +1 traduit la présence d'un photon Stokes spontané dans ce même mode.

En faisant l'hypothèse que les coefficients d'atténuations linéiques ne sont pas dispersifs $\left(\alpha_{\mathrm{p}} \simeq \alpha_{\mathrm{s}} \simeq \alpha\right)$, on aboutit aux équations couplées :

$$
\begin{aligned}
& \frac{\mathrm{d} n_{\mathrm{p}}}{\mathrm{d} Z}+n_{\mathrm{p}}\left(\alpha+\gamma_{\mathrm{s}} n_{0} \mathrm{e}^{-\alpha Z}+q \gamma_{\mathrm{s}}\right)=\gamma_{\mathrm{s}} n_{\mathrm{p}}^{2} \\
& \frac{\mathrm{d} n_{\mathrm{s}}}{\mathrm{d} Z}+n_{\mathrm{s}}\left(\alpha+\gamma_{\mathrm{s}} q-\gamma_{\mathrm{s}} n_{0} \mathrm{e}^{-\alpha Z}\right)=-\gamma_{\mathrm{s}} n_{\mathrm{s}}^{2}+q \gamma_{\mathrm{s}} n_{0} \mathrm{e}^{-\alpha Z}
\end{aligned}
$$

obtenues à partir des conditions initiales en $Z=0$ :

$$
\begin{aligned}
& n_{\mathrm{s}}=\sum_{j=1}^{q} n_{\mathrm{s} j} \\
& n_{\mathrm{p}}(Z=0)=n_{\mathrm{p}}^{0} \\
& n_{\mathrm{s}}(Z=0)=\sum_{j=1}^{q} n_{\mathrm{s} j}^{0}=n_{\mathrm{s}}^{0}
\end{aligned}
$$

la conservation du nombre total de photons étant donnée par :

$$
n_{\mathrm{s}}+n_{\mathrm{p}}=n_{0} \mathrm{e}^{-\alpha Z}
$$

où

$$
n_{0}=n_{\mathrm{p}}^{0}+n_{\mathrm{s}}^{0}
$$

représente le nombre total de photons incidents en $Z=0$ et $q$ le nombre de modes longitudinaux situés dans la courbe de gain Raman. La résolution de ces deux équations couplées aboutit à la solution en régime quasi stationnaire :

$$
\begin{aligned}
& n_{\mathrm{s}}=n_{0} \mathrm{e}^{-\alpha Z} \frac{(A+B)}{(1+A+B)} \\
& n_{\mathrm{p}}=n_{0} \mathrm{e}^{-\alpha Z}(1+A+B)^{-1}
\end{aligned}
$$

avec :

$$
\begin{aligned}
& A=\frac{n_{\mathrm{s}}^{0}}{n_{\mathrm{p}}^{0}} \exp \left(q \gamma_{\mathrm{s}} Z+\frac{\gamma_{\mathrm{s}} n_{0}}{\alpha}\left(1-\mathrm{e}^{-\alpha Z}\right)\right) \\
& B=\frac{q}{n_{\mathrm{s}}^{0}} A .
\end{aligned}
$$

La conversion d'un nombre de photons en puissance optique s'effectue à travers l'égalité :

$$
\gamma_{\mathrm{s}} n_{\mathrm{p}}^{0}=g_{\mathrm{R}} \frac{P_{\mathrm{p}}^{0}}{A_{\mathrm{eff}}} .
$$

$P_{\mathrm{p}}^{0}$ correspond à la puissance de pompe couplée à la fibre et $A_{\text {eff }}$ à une aire effective fonction de la répartition spatiale du mode transverse.

Dans l'approximation paramétrique et en considérant $n_{\mathrm{p}}^{0} \gg n_{\mathrm{s}}^{0}$, les équations couplées (1) (2) s'écrivent :

$$
\begin{aligned}
& \left(\frac{\mathrm{d}}{\mathrm{d} Z}+\alpha_{\mathrm{s} j}\right) P_{\mathrm{s} j}(Z)=g_{\mathrm{R} j} \frac{P_{\mathrm{p}}(Z)}{A_{\mathrm{eff}}} P_{\mathrm{s} j}(\mathrm{Z}) \\
& \left(\frac{\mathrm{d}}{\mathrm{d} Z}+\alpha_{\mathrm{p}}\right) P_{\mathrm{p}}(Z)=0
\end{aligned}
$$

dont les solutions classiques sont du type :

$$
\begin{aligned}
& P_{\mathrm{p}}(Z)=P_{\mathrm{p}}^{0} \exp \left(-\alpha_{\mathrm{p}} Z\right) \\
& P_{\mathrm{s} j}(Z)=P_{\mathrm{s} j}^{0} \exp \left(-\alpha_{\mathrm{s} j} Z+g_{\mathrm{R} j} \frac{P_{\mathrm{p}}^{0}}{A_{\mathrm{eff}}} L_{\text {eff }}\right) .
\end{aligned}
$$
pompe

$L_{\text {cff }}$ correspond à une longueur effective d'interaction prenant en compte l'affaiblissement de l'onde de

$$
L_{\text {eff }}=\frac{1-\exp \left(-\alpha_{\mathrm{p}} Z\right)}{\alpha_{\mathrm{p}}}
$$


Cette longueur effective d'interaction tend vers $1 / \alpha_{\mathrm{p}}$ et elle sera d'autant plus grande que le coefficient d'atténuation à la longueur d'onde de pompe $\lambda_{\mathrm{p}}$ sera faible.

En l'absence d'onde signal sur la fréquence Stokes, un processus de transfert de l'onde de pompe vers l'onde Stokes peut exister, traduisant l'amplification de photons Stokes spontanés. Cette émission peut être déterminée en sommant sur l'ensemble des modes longitudinaux, la puissance optique contenue dans la courbe de gain Raman spontané du milieu

$$
P_{\mathrm{s}}(Z)=\int_{\substack{\text { modes } \\ \text { longitudinaux }}} h \nu_{\mathrm{s} j} \exp \left(-\alpha_{\mathrm{s} j} Z+g_{\mathrm{R} j}(\nu) \frac{P_{\mathrm{p}}^{0}}{A_{\text {eff }}} L_{\text {eff }}\right) \mathrm{d} \nu .
$$

La courbe de gain Raman peut être approximée par une loi de distribution du type Lorentzienne [17] :

$$
g_{\mathrm{R}}(\nu) \simeq g_{\mathrm{R}}\left(1-\left(\frac{\nu-\nu_{\mathrm{R}}}{\frac{\Delta \nu_{\mathrm{R}}}{2}}\right)^{2}\right)
$$

où $\nu_{\mathrm{R}}$ et $\Delta \nu_{\mathrm{R}}$ correspondent respectivement à la fréquence centrale de cette raie et à la largeur spectrale à mi-hauteur. Dans ces conditions, l'expression (19) s'écrit :

$$
P_{\mathrm{s}}(Z)=h \nu_{\mathrm{s}} \exp \left(-\alpha_{\mathrm{s}} Z+P_{\mathrm{p}}^{0} \frac{L_{\mathrm{eff}}}{A_{\text {eff }}} g_{\mathrm{R}}\right) B_{\text {eff }} .
$$

La bande de fréquence effective du gain étant :

$$
B_{\text {eff }}=\frac{\sqrt{\pi}}{2} \frac{\Delta \nu_{\mathrm{R}}\left(A_{\mathrm{eff}}\right)^{1 / 2}}{\left(P_{\mathrm{p}}^{0} L_{\mathrm{eff}} g_{\mathrm{R}}\right)^{1 / 2}}
$$

et la puissance Stokes fictive en $Z=0$ en l'absence d'une onde signal devient :

$$
P_{\mathrm{B}}^{0}=h \nu_{\mathrm{s}} B_{\mathrm{eff}}=\sum P_{\mathrm{s} j}^{0} .
$$

Cette puissance optique correspond à une puissance de bruit à l'entrée de la fibre et le nombre de modes longitudinaux $q$ sur lesquels peut se développer le processus non linéaire, est donné par la relation :

$$
q=\frac{B_{\text {eff }}}{\Delta \nu}=B_{\text {eff }} \frac{n_{\text {seff }} L}{c}
$$

$c$ étant la vitesse de la lumière dans le vide, $n_{\text {seff }}$, l'indice effectif du mode à la fréquence Stokes, $\Delta \nu$ caractérisant l'écartement spectral entre modes longitudinaux adjacents en régime de superradiance.

Dans l'approximation gaussienne des champs [18] associés au mode fondamental aux fréquences pompe $\omega_{\mathrm{p}}$ et Stokes $\omega_{\mathrm{s}}$,

$$
\left(E_{x}^{\omega_{\mathrm{p}}} \propto \exp \left(-\left(\frac{x}{W_{0 \mathrm{p}}}\right)^{2}\right) E_{x}^{\omega_{\mathrm{s}}} \propto \exp \left(-\left(\frac{x}{W_{0 \mathrm{~s}}}\right)^{2}\right)\right)
$$

l'aire effective s'écrit simplement :

$$
A_{\text {eff }} \simeq \pi W_{0_{\text {eff }}}^{2}=\pi \frac{\left(W_{0 \mathrm{p}}^{2}+W_{0 \mathrm{~s}}^{2}\right)}{2}
$$

$W_{0 \mathrm{p}}$ et $W_{0 \mathrm{~s}}$ étant les rayons du mode aux longueurs d'onde pompe et Stokes.

Si l'on prend en considération un effet de « déplétion » de l'onde de pompe sous l'effet du transfert d'énergie, on aboutit aux solutions $[16,19]$ :

$$
\begin{gathered}
P_{\mathrm{p}}(Z)=P_{0} \exp (-\alpha Z)(1+A+B)^{-1} \\
P_{\mathrm{s}}(Z)=\frac{\omega_{\mathrm{s}}}{\omega_{\mathrm{p}}} P_{0} \exp (-\alpha Z) \times \\
\times(A+B)(1+A+B)^{-1} \\
P_{0}=P_{\mathrm{p}}^{0}+\frac{\omega_{\mathrm{p}}}{\omega_{\mathrm{s}}} P_{\mathrm{s}}^{0} .
\end{gathered}
$$

Le gain d'amplification est alors déterminé en effectuant le rapport entre l'amplitude du signal mesurée en présence de l'onde de pompe et celle obtenue en l'absence de cette même onde de pompe à l'entrée de la fibre :

$$
G(\mathrm{~dB})=10 \log \left\{\frac{P_{\mathrm{s}}(Z) \rightarrow P_{\mathrm{p}}^{0} \neq 0}{P_{\mathrm{s}}(Z) \rightarrow P_{\mathrm{p}}^{0}=0}\right\} .
$$

Le rapport signal sur bruit est défini comme étant le rapport entre l'amplitude du signal mesurée à la fréquence Stokes en présence d'une onde signal couplée à la fibre en $Z=0$ et celle détectée en l'absence de cette même onde signal :

$S / B(\mathrm{~dB})=10 \log \left\{\frac{P_{\mathrm{s}}(Z) \rightarrow P_{\mathrm{s}}^{0} \neq 0}{P_{\mathrm{s}}(Z) \rightarrow P_{\mathrm{s}}^{0}=0}\right\}$.

Le bruit correspondant dans ce cas à l'amplification des photons Stokes spontanés. 
3.1 MONTAGE EXPÉRIMENTAL. - Le montage décrivant ce type d'interaction pour l'amplification optique de signaux est schématisé sur la figure $1[20$, 21]. On couple à une fibre optique unimodale une onde de pompe à la fréquence angulaire $\omega_{\mathrm{p}}$ et une onde signal $\omega_{s}$; l'espacement en fréquence entre ces deux ondes correspondant au décalage Raman $\left(\omega_{\mathrm{p}}-\omega_{\mathrm{s}}=\omega_{\mathrm{R}}\right)$, caractéristique du milieu. Pour la silice faiblement dopée $450<\frac{\omega_{\mathrm{R}}}{2 \pi C}<490 \mathrm{~cm}^{-1}$.

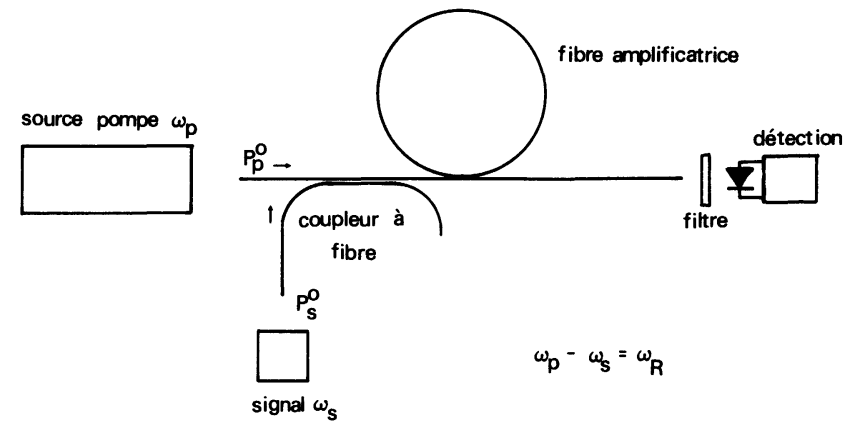

Fig. 1. - Amplification Raman (Schéma synoptique).

[Raman amplification (experimental set-up).]

Un filtre spectral placé devant le photodétecteur élimine la composante $\omega_{\mathrm{p}}$ et présente un maximum de transmission à $\omega_{\mathrm{s}}$.

3.2 RÉSULTATS EXPÉRIMENTAUX. - Un relevé typique donnant l'évolution du gain d'amplification d'un signal émis par une diode laser $\left(\lambda_{\mathrm{s}}=1,318 \mu \mathrm{m}\right)$ en fonction de la puissance de pompe crête $\left(\lambda_{p}=1,238 \mu \mathrm{m}\right)$ est présenté sur la figure $2 a$.

Les courbes calculées à partir des caractéristiques optogéométriques de la fibre amplificatrice sont également rapportées. Deux types d'interaction sont à considérer dans la modélisation, le premier cas correspond à une interaction optimisée où les ondes de pompe et Stokes sont dans un état de polarisations rectilignes et parallèles (courbe - - ).

Si la fibre n'est pas à maintien de polarisation, l'interaction peut être décrite par un processus d'échange d'énergie via la non-linéarité Raman avec états de polarisation brouillés entre ondes (courbe -. - -).

On peut également modéliser et comparer les caractéristiques de bruit associées à ce type d'amplification optique. C'est ce que nous avons représenté sur la figure $2 \mathrm{~b}$ où l'on peut comparer les résultats issus d'une mesure et ceux provenant d'une modélisation.

Les courbes donnant l'évolution du gain d'amplification (Fig. 2a) sont caractérisées par deux régimes.

Une première région correspond à un gain variant linéairement en $\mathrm{dB}$ avec la puissance de pompe,

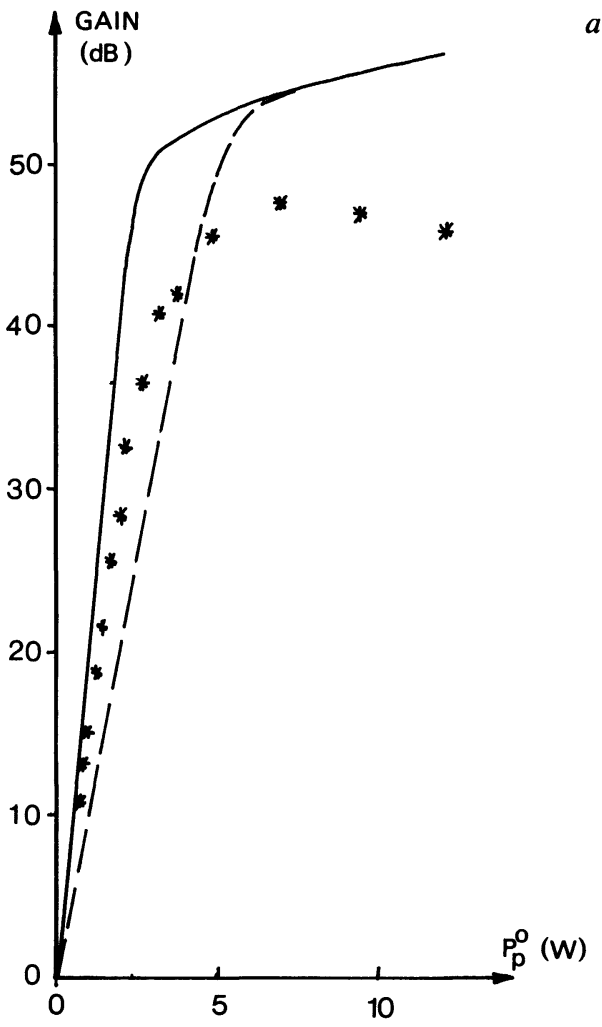

a)

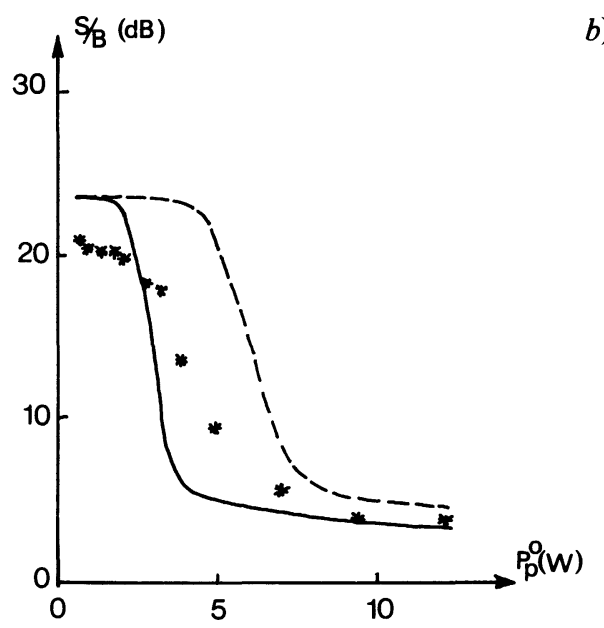

Fig. 2. - a) Evolution du gain d'amplification en fonction de la puissance de pompe crête $\left(\lambda_{\mathrm{p}}=1,24 \mu \mathrm{m}\right.$, $\lambda_{\mathrm{s}}=1,318 \mu \mathrm{m}, \quad P_{\mathrm{s}}^{0}=23,4 \mu \mathrm{W}, \quad L=2,8 \mathrm{~km}$, $\left.W_{\text {eff }}^{0}=3,17 \mu \mathrm{m}\right){ }^{*}$ points expérimentaux ; — courbes calculées. b) Caractéristiques du rapport $\mathrm{S} / \mathrm{B}$ en fonction de la puissance de pompe crête. - interaction optimisée (conservation des états de polarisations parallèles entre $\omega_{\mathrm{p}}$ et $\left.\omega_{\mathrm{s}}\right)$; --.- interaction avec états de polarisations brouillés.

[a) Amplification gain dependence on peak pump power $\left(\lambda_{\mathrm{p}}=1.24 \mu \mathrm{m}, \quad \lambda_{\mathrm{s}}=1.318 \mu \mathrm{m}, \quad P_{\mathrm{s}}^{0}=23.4 \mu \mathrm{W}\right.$, $\left.L=2.8 \mathrm{~km}, W_{\text {eff }}^{0}=3.17 \mu \mathrm{m}\right) . \quad *$ experimental points ; -.. calculated curves. b) Signal to noise ratio versus peak pump power - optimized interaction assuming that polarization between the pump and signal waves are parallels ; --- corresponding to scrambled polarization case.] 
suivi d'une saturation traduisant l'amplification des photons Stokes spontanés et l'apparition d'un processus multistokes. Dans ce dernier cas, l'onde amplifiée se comporte à son tour comme une nouvelle onde de pompe et un transfert de puissance peut s'opérer vers l'onde de fréquence Stokes qui lui est adjacente.

Des gains d'amplification compris entre 30 et $50 \mathrm{~dB}$ ont été mesurés, ces coefficients d'amplification étant dépendants de la dimension du mode de la fibre, de l'atténuation spectrale, de la longueur d'interaction et de l'amplitude du signal couplée dans la fibre à la fréquence Stokes.

Sur la figure $2 a$, la pente différentielle du gain mesurée est de l'ordre de $15 \mathrm{~dB} / \mathrm{W}$ pour une fibre longue de $2,8 \mathrm{~km}$ et caractérisée par une dimension de mode de $3,2 \mu \mathrm{m}$.

3.3 APPliCATIONS. - L'effet Raman stimulé dans les fibres optiques représente une limitation dans les systèmes de Télécommunications quant au niveau de puissance optique pouvant être couplé. Son utilisation avec un schéma d'interaction approprié permet néanmoins d'effectuer des opérations d'amplification de signaux par voie purement optique. Cette amplification exploitant l'aspect résonnant du matériau permet ainsi d'accroître la portée d'un système dans le domaine des Télécommunications. Le traitement optique du signal peut également utiliser ce type d'interactions non linéaires (corrélation temporelle, ...). Dans cet ordre d'idées, la réalisation de lignes à retard caractérisées par un fort produit $B . \tau$ peut être effective avec l'emploi de fibres optiques unimodales, par recirculation d'une impulsion dans une boucle à fibre. $\tau$ représente dans ce cas, le temps de stockage d'une impulsion et $B$ (bande passante du système) est reliée aux caractéristiques de dispersion de cette impulsion dans le milieu.

On exploite dans ce cas une des principales propriétés des fibres optiques unimodales qui repose sur la très faible dispersion des signaux sur de grandes distances [22]. Dans la configuration à boucle avec recirculation d'une impulsion, cette dernière se trouve être atténuée en fonction du nombre de tours effectués. Afin de compenser cet affaiblissement, il a été démontré que la régénération de cette impulsion pouvait être réalisée en utilisant l'amplification Raman [23]. Les applications de ce type de systèmes à boucles entrent naturellement dans le domaine du traitement de signaux radar et offrent par ailleurs d'intéressantes perspectives dans le domaine des gyromètres à fibres [24].

\section{Mélange à quatre photons.}

Un autre mécanisme rendant compte de l'effet Kerr ou d'opérations de mélange de fréquence peut se développer dans les matériaux de type centro-symétriques. Dans ce cas, la non-linéarité optique émane d'une contribution électronique (partie réelle de $\left.\chi^{(3)}\right)$. Elle est de plus, de forte amplitude mais nécessite la réalisation d'un accord de phase. La description phénoménologique de cette interaction non linéaire du troisième ordre, de type mélange de fréquences, repose sur l'annihilation de deux photons d'une onde de pompe pour créer un photon à la fréquence antistokes $\left(\omega_{\mathrm{AS}}>\omega_{\mathrm{p}}\right)$ et un photon à la fréquence Stokes $\left(\omega_{\mathrm{s}}<\omega_{\mathrm{p}}\right)$. On emploie cette dénomination anti-Stokes et Stokes (idler et signal) par analogie avec une interaction de type Raman.

L'équation différentielle gouvernant l'évolution des champs dans un milieu non linéaire de structure guide optique, de configuration cylindrique et homogène s'écrit $[25,26]$ :

$$
\begin{aligned}
& \frac{\mathrm{d}}{\mathrm{d} Z} A_{\nu}^{(i)}(Z)+\frac{\alpha_{i}}{2} A_{\nu}^{(i)}= \\
& \quad=j \frac{\omega_{i}}{4} \iint P_{\mathrm{NL}} E_{\nu}^{(i)^{*}} \mathrm{e}^{-j \beta(i)} \nu z r \mathrm{~d} r \mathrm{~d} \theta
\end{aligned}
$$

$P_{\mathrm{NL}}$ représente le terme de polarisation non linéaire.

On considère que le champ total est une superposition d'ondes planes se propageant dans la direction $\mathrm{OZ}$. Ces champs sont polarisés linéairement dans une même direction et la composante scalaire peut se mettre sous la forme :

$$
E(Z, r, \theta, t)=\sum_{i} \sum_{\nu} A_{\nu}^{(i)}(Z) \mathrm{e}^{j\left(\beta_{\nu}^{(i)} Z-\omega_{i} t\right)} E_{\nu}^{(i)}(r, \theta)
$$

$i$ représente les différents champs de fréquence $\omega_{i}$ et $\nu$ les différents modes sur lesquels ils sont distribués. $E_{\nu}(r, \theta)$ est associé à la répartition spatiale du champ considéré comme quasi transverse et $\beta_{\nu}$ représente la constante de propagation longitudinale caractérisant le mode d'ordre $\nu$.

En régime de propagation monomode, les équations différentielles rendant compte de l'évolution des champs aux fréquences Stokes et anti-Stokes dans l'approximation paramétrique pour l'onde de pompe, s'écrivent :

$$
\begin{aligned}
& \frac{\mathrm{d}}{\mathrm{d} Z} A_{\mathrm{s}}+\frac{\alpha_{\mathrm{s}}}{2} A_{\mathrm{s}}=j \frac{\omega_{\mathrm{s}}}{4}\left(a \chi_{\mathrm{ss}} A_{\mathrm{s}}+c \exp (j \Delta \beta Z) \chi_{\mathrm{sa}} A_{\mathrm{a}}^{*}\right) \\
& \frac{\mathrm{d}}{\mathrm{d} Z} A_{\mathrm{a}}^{*}+\frac{\alpha_{\mathrm{a}}}{2} A_{\mathrm{a}}^{*}=-j \frac{\omega_{\mathrm{a}}}{4}\left(b \chi_{\mathrm{aa}}^{*} A_{\mathrm{a}}^{*}+c^{*} \exp (-j \Delta \beta . Z) \chi_{\mathrm{sa}} A_{\mathrm{s}}\right)
\end{aligned}
$$

où : $\chi_{\mathrm{ss}}, \chi_{\mathrm{aa}}$ et $\chi_{\mathrm{sa}}$ représentent les susceptibilités non linéaires liées respectivement aux interactions onde pompe-onde Stokes, onde pompe-onde anti-Stokes, onde Stokes-onde anti-Stokes. 
Les facteurs $a, b, c$ correspondent aux intégrales de recouvrement entre les champs des ondes pompe Stokes et anti-Stokes :

$$
\begin{aligned}
& a=|A p|^{2} \cdot\langle s, p, p, s\rangle \\
& b=A p^{2} \cdot\langle s, p, p, a\rangle \\
& c=|A p|^{2} \cdot\langle a, p, p, a\rangle .
\end{aligned}
$$

Les termes entre crochets, étant associés aux opérations :

$$
\langle i, j, k, l\rangle=\frac{\left\langle E_{i} E_{j} E_{k} E_{l}\right\rangle}{\left\langle E_{i}^{2}\right\rangle^{1 / 2}\left\langle E_{j}^{2}\right\rangle^{1 / 2}\left\langle E_{k}^{2}\right\rangle^{1 / 2}\left\langle E_{l}^{2}\right\rangle^{1 / 2}}
$$

et ont pour grandeur, l'inverse d'une surface effective, avec :

$$
\left\langle E_{i}^{2}(r, \theta)\right\rangle=\int_{0}^{\infty} \int_{0}^{2 \pi} E_{i}^{2}(r, \theta) r \mathrm{~d} r \mathrm{~d} \theta
$$

$\Delta \beta$ représente le désaccord de phase :

$$
\Delta \beta=2 \beta_{\nu}^{\mathrm{p}}-\beta_{\nu}^{\mathrm{a}}-\beta_{\nu}^{\mathrm{s}}
$$

$\boldsymbol{\beta}_{\nu}{ }^{(i)}$ représentant la constante de propagation du mode $\nu$ à la fréquence pompe, anti-Stokes et Stokes $(i=p, a, s)$.

Dans le cas où l'on néglige l'atténuation différentielle et en faisant l'hypothèse qu'en $z=0, A_{\mathrm{a}}^{0}=0$, la résolution des équations (33), (34) aboutit aux solutions en termes de puissances optiques :

$$
\begin{gathered}
P_{\mathrm{s}}=P_{\mathrm{s}}^{0}\left(1+\frac{\left(g_{0} Z\right)^{2}}{4} \frac{\sinh ^{2}\left(g_{\mathrm{M} 4 \mathrm{P}} Z\right)}{\left(g_{\mathrm{M} 4 \mathrm{P}} \cdot Z\right)^{2}}\right) \\
P_{\mathrm{a}}=P_{\mathrm{s}}^{0} \frac{\sinh ^{2}\left(g_{\mathrm{M} 4 \mathrm{P}} \cdot Z\right)}{\left(g_{\mathrm{M} 4 \mathrm{P}} \cdot Z\right)^{2}}
\end{gathered}
$$

avec :

$$
\begin{gathered}
g_{0}=P_{\mathrm{p}}^{2}\langle s, p, p, a\rangle^{2}\left(\frac{\chi^{1(3)} \omega_{\mathrm{p}}}{2}\right)^{2} \\
g_{\mathrm{M} 4 \mathrm{P}}=\left(g_{0}^{2}-\left(\frac{\Delta \beta}{2}\right)^{2}\right)^{1 / 2}
\end{gathered}
$$

$g_{\mathrm{M} 4 \mathrm{P}}$ représente le coefficient de gain spécifique au processus de mélange à quatre photons. Pour les fréquences Stokes et anti-Stokes, le gain sera par ailleurs d'autant plus grand que le terme de désaccord de phase sera faible.

4.1 CONDITIONS D'ACCORD DE PHASE DANS LES FIBRES OPTIQUES. - Le terme décrivant le désaccord de phase $\Delta \beta$ a deux contributions, l'une liée à la dispersion du matériau $\left(\Delta \beta_{\mathrm{m}}\right)$ et la seconde associée à la dispersion des modes dans la structure de guidage $\left(\Delta \beta_{\mathrm{g}}\right)$. A ces conditions, se superpose la règle de conservation de l'énergie qui se traduit par la relation:

$$
2 \omega_{\mathrm{p}}-\omega_{\mathrm{s}}-\omega_{\mathrm{a}}=0 .
$$

Lorsque l'on évalue la dépendance du terme $\Delta \beta_{\mathrm{m}}$ en fonction du décalage en fréquence $\Delta \nu$ par rapport à la longueur d'onde de pompe $\lambda_{\mathrm{p}}$ située à $1,32 \mu \mathrm{m}$ (au voisinage de la longueur d'onde de dispersion chromatique nulle pour une fibre optique à base de silice), nous obtenons la courbe présentée sur la figure 3a. Selon les paramètres optogéométriques de la fibre, la seconde contribution suit une dépendance avec le décalage en fréquence donnée par la figure $3 b$. Le désaccord de phase total est représenté par la somme des deux effets, ce que nous avons représenté sur la figure 4.

Ainsi, en modifiant le rayon de cœur d'une fibre, durant l'opération de fibrage par exemple, il est possible d'ajuster les conditions d'accord de phase ou d'annuler $\Delta \beta$ pour deux longueurs d'ondes données $\lambda_{\mathrm{s}}$ et $\lambda_{\mathrm{as}}$.

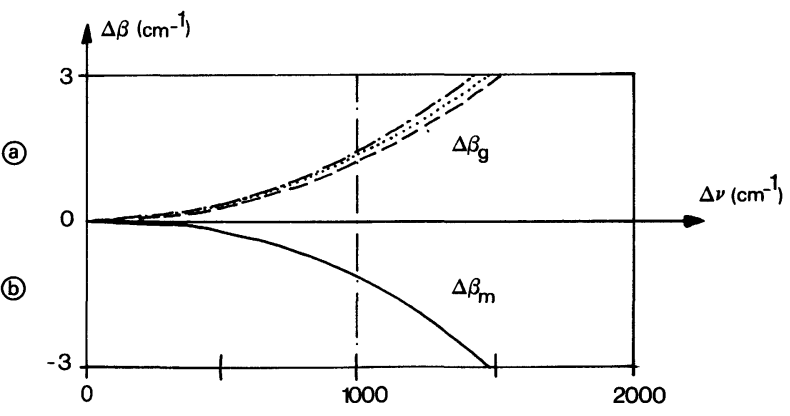

Fig. 3. - Evolution du désaccord de phase en fonction du décalage en fréquence. a) Contribution due à la dispersion du mode. b) Contribution liée à la dispersion de l'indice, $\lambda_{\mathrm{p}}=1,319 \mu \mathrm{m}$, différence d'indice $\Delta n=3,87 \times 10^{-3}$, rayon de cour : $a=4,15 \mu \mathrm{m}$ (-. ) $a=4,224 \mu \mathrm{m}$ (...), $a=4,33 \mu \mathrm{m}$ (----).

[Phase mismatch versus frequency shift $\Delta \nu$ as a function of fiber's parameters. The pump is fixed at $1.319 \mu \mathrm{m}$. a) Phase mismatch due to waveguide dispersion. b) Phase mismatch due to material dispersion, $\lambda_{p}=1.319 \mu \mathrm{m}$, index difference $\Delta n=3.87 \times 10^{-3}$, core radius :

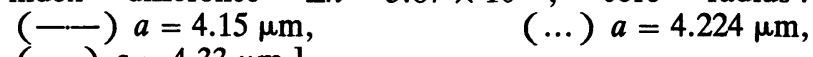
(---) $a=4.33 \mu \mathrm{m}$. 


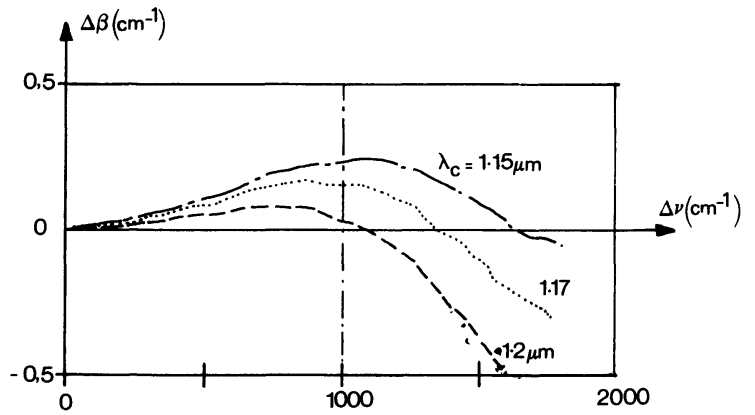

Fig. 4. - Désaccord de phase total en fonction des paramètres optogéométriques des fibres. $\lambda_{\mathrm{c}} \simeq 2 a \sqrt{n_{1} 2 \Delta n} 2,612 . \quad \lambda_{\mathrm{c}}=$ longueur d'onde à partir de laquelle la fibre optique présente le caractère unimodal.

[Phase mismatch versus the optogeometrical parameters of the monomode fiber. $\lambda_{\mathrm{c}}=2 a \sqrt{n_{1} 2 \Delta n} \times 2.612$. $\lambda_{c}=$ cutoff wavelength of the LP11 modes.]

Ainsi, pour ces deux longueurs d'ondes, un processus d'échange d'énergie efficace pourra être observé, provenant d'une onde de pompe $\lambda_{\mathrm{p}}$.

4.2 RÉSUltATS EXPÉRIMENTAUX. - Nous allons illustrer cet effet en considérant une onde de pompe émise par un laser YAG opérant à $\lambda_{\mathrm{p}}=1,319 \mu \mathrm{m}$ et travaillant en régime déclenché. Le faisceau issu de cette source est couplé à une fibre optique monomode longue de $20 \mathrm{~m}$, réalisée au Laboratoire Fibres Optiques du LCR.

Lorsque l'on analyse le spectre émis par cette fibre excitée par une onde de pompe dont la puissance crête vaut $150 \mathrm{~W}$, on observe deux raies (Fig. 5) caractéristiques de la génération de nouvelles fréquences dues au rayonnement de la polarisation non linéaire induite par l'onde de pompe. Les conditions

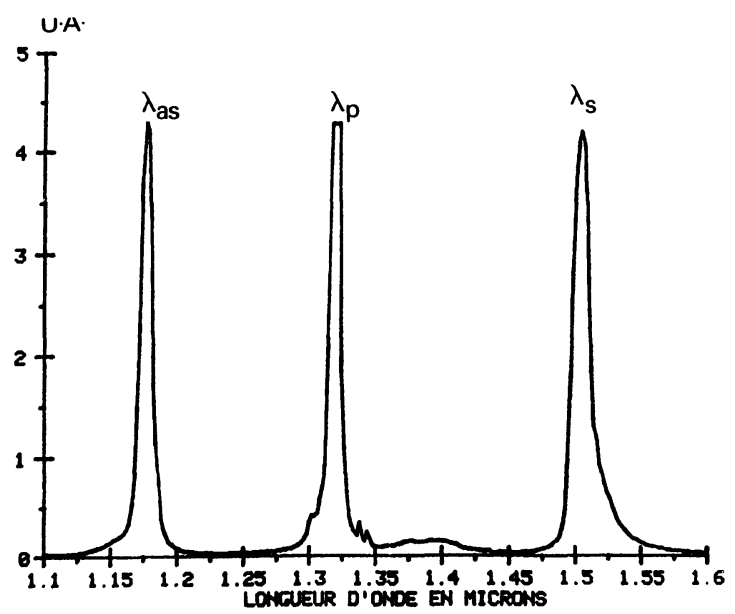

Fig. 5. - Spectre mesuré en sortie de fibre $(L=20 \mathrm{~m})$ caractéristique d'une opération de mélange de fréquences $\left(\lambda_{\mathrm{p}}=1,319 \mu \mathrm{m}\right)$.

[Typical output spectra measured in phase matched stimulated four photons mixing.] d'accord de phase sont alors respectées pour ces deux fréquences $\omega_{\text {s }}$ et $\omega_{\text {as }}$.

Si maintenant la fibre optique est placée dans une étuve, en variant la température de la fibre, on modifie les conditions d'accord de phase, ce qui se traduit par un changement de position des raies Stokes et anti-Stokes générées par mélange de fréquences [26].

Sur la figure 6 , sont rapportés les spectres obtenus en bout de fibre lorsque celle-ci voit sa température modifiée, passant de 29 à 113 degrés centigrades. Les ondes Stokes et anti-Stokes correspondant aux raies situées respectivement au voisinage de 1,18 et $1,49 \mu \mathrm{m}$ voient leurs positions se déplacer sous l'effet de la température. Cette observation traduisant un contrôle ou un ajustement des conditions d'accord de phase ouvre donc la voie à la réalisation de sources accordables dans la fenêtre $1-1,6 \mu \mathrm{m}$ à partir : d'une source de pompe $\left(\lambda_{p}=1,319 \mu \mathrm{m}\right)$, d'une fibre optique unimodale et d'une étuve. La réalisation de ce type de source a bien des applications dans le domaine de la caractérisation de composants optoélectroniques. Des opérations d'amplification optique de signaux ou de conversion de fréquences ont également été démontrées à partir de ce type d'interaction non linéaire [27].

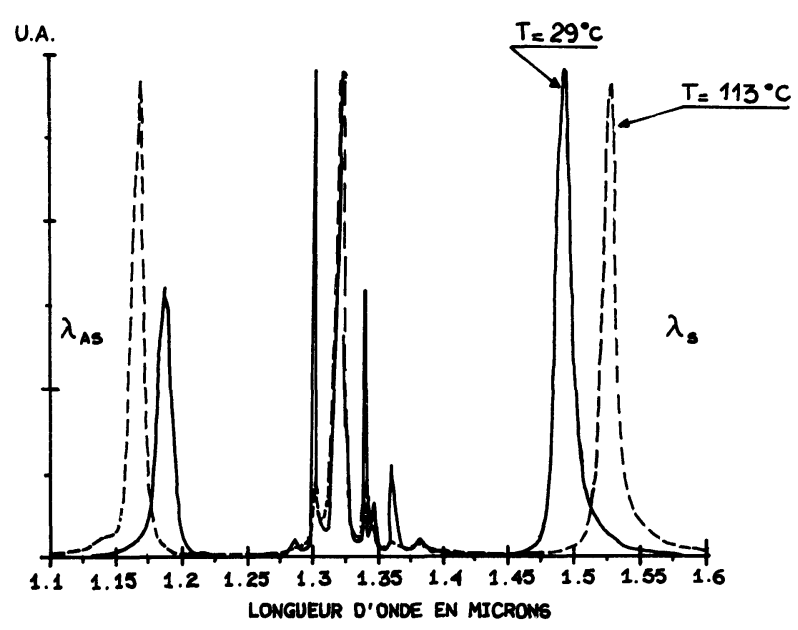

Fig. 6. - Effet de la température sur la génération d'ondes Stokes et signal par mélange de trois ondes dans une fibre optique unimodale.

[Temperature frequency tuning on Stokes and signal wavelengths based on stimulated four photons mixing in monomode fiber.]

\section{Conclusion.}

Nous avons montré dans cet article que la configuration guide optique permet d'entrevoir la réalisation de nouveaux composants exploitant les effets non linéaires. Son extension dans le domaine des nonlinéarités du second ordre est évidente, en particulier avec l'emploi de matériaux cristallins [19] ou organiques [15]. 
Afin d'illustrer les possibilités offertes par ces interactions de type non linéaires dans les guides optiques, nous avons décrit deux effets observés dans les fibres optiques à base de silice et leurs exploitations.

En fait, l'emploi de ces mécanismes dans des systèmes impose l'utilisation de faibles niveaux de puissance de commande (i.e. pompe). Nous avons montré par exemple, que l'effet Raman stimulé dans les fibres optiques satisfait à ce critère. Le développement de nouveaux matériaux à forts coefficients non linéaires $\left(\chi^{(2)}, \chi^{(3)}\right)$ conditionnés sous forme de guides optiques ouvre la voie à la conception dans le domaine du traitement optique de l'information, de fonctions adaptées (amplification, conversion de fréquences, portes optiques logiques, bistabilité intrinsèque, corrélation optique, ...). Parallèlement, la réalisation de sources optiques de type diodes laser à structures couplées [28] délivrant des puissances optiques de l'ordre du Watt ne peut qu'accélérer le développement de tels systèmes.

\section{Remerciements.}

Nous remercions les Laboratoires Semi-conducteurs pour Optoélectronique et Fibres Optiques du LCR pour la réalisation des composants utilisés dans nos expériences. Cette étude a été soutenue en partie, par un contrat CNET DAII.

\section{Bibliographie}

[1] Snitzer, E., J. Appl. Phys. 32 (1961) 36-39.

[2] Koester, C. J., Snitzer, E., Appl. Optics 3 (1964) 1182-1186.

[3] Ross, B., SNITZER, E., IEEE J. Quantum Electron. QE-6 (1970) 361-366.

[4] Holst, G. C., Snitzer, E., IEEE Conf. on Laser Eng. and Applications, 1969, CLEA 1969.

[5] Snitzer, E., J. Opt. Soc. Am. 51 (1961) 491-498.

[6] Snitzer, E., Osterberg, H., J. Opt. Soc. Am. 51 (1961) 499-505.

[7] Miya, V., Terunuma, Y., Hosaka, T., Miyashita, T., Elect. Lett. 15 (1979) 106-108.

[8] Blankenship, M. G., DenEKA, C. W., IEEE J. Quantum Electron. QE-18 (1982) 1418-1423.

[9] Poole, S. B., Payne, D. N., Fermann, M. E., Electron. Lett. 21 (1985) 737-738.

[10] Reekie, L., Mears, R. J., Payne, D. N., Poole, S. B., IOOC-ECOC'85, Venizia, Oct 1-4, 1985.

[11] Fejer, M., Byer, R. L., Feigelson, R., KwAy, W., Proc. SPIE, vol. 320, Adv. Infrared Fibers II (1982) Los Angeles.

[12] Digonnet, M. J. F., Shaw, H. J., II ECIO Firenze 17-18/10/1983.

[13] Magel, G. A., Fejer, M. M., Nightingale, J. L., BYER, R. L., CLEO'85, Baltimore 23 May 1985.

[14] Zyss, J., LedouX, I., Hierle, R. B., RAJ, R. K., OUDAR, J. L., IEEE J. Quantum Electron. QE21 (1985) 1286.
[15] LedouX, I., Vidakovic, P., Zyss, J., Colloque National Matériaux II, MRT/CNRS, Paris 2729/1/86.

[16] AuYeung, J., Yariv, A., IEEE J. Quantum Electron. QE-14 (1978) 347.

[17] Sмith, R. G., Appl. Opt. 11 (1972) 2489.

[18] Marcuse, D., Bell Syst. Tech. J. 56 (1977) 703.

[19] Desurvire, E., Pocholle, J. P., RafFy, J., PaPuCHON, M., L'Optique guidée monomode et ses applications (Masson) 1985.

[20] Desurvire, E., Papuchon, M., Pocholle, J. P., RAFFY, J., OSTROWSKY, D. B., Electron. Lett. 19751.

[21] Pocholle, J. P., Raffy, J., Papuchon, M., DesurVIRE, E., Opt. Eng. 24 (1985) 600.

[22] KorotKI, S. K., et al. O.F.C.'85 San Diego (Feb. 1113, 1985) post deadline paper.

[23] Desurvire, E., Digonnet, M., Shaw, H. J., Optics Lett. 10 (1985) 83.

[24] Nakazawa, M., Opt. Lett. 10 (1985) 193.

[25] Saissy, A., Botineau, J., Azema, A., Gires, F., Appl. Optic. 19 (1980) 1639.

[26] Stolen, R. H., BJorkholm, J. E., IEEE J. Quantum Electron. 18 (1982) 1062.

[27] Pocholle, J. P., Raffy, J., Papuchon, M., OFC'85, San Diego (Feb. 11-13, 1985).

[28] Scifres, D. R., Streifer, W., Burnham, R. D., Lindstrom, C., PAOLI, T., IOOC'83, Tokyo (June 27-30, 1983). 